\title{
Renal vein thrombosis in cases of polyarteritis nodosa and of the Henoch-Schoenlein syndrome
}

\author{
M. E. J. BEARD AND D. J. E. TAYLOR \\ From the Group Pathology Laboratory, St Stephens Hospital, London
}

SYNOPSIS The occurrence of renal vein thrombosis in a case each of polyarteritis nodosa and the Henoch-Schoenlein syndrome is reported. It is suggested that obliteration of the arterioles than supply the walls of the veins may have initiated the venous thrombosis in these two cases.

Renal vein thrombosis has been divided into four main groups (Harrison, Milne, and Steiner, 1956): it may be secondary to thrombosis or obstruction of the inferior vena cava; it may occur as a primary renal vein thrombosis; or it may complicate renal disease. Many renal diseases have been associated with renal vein thrombosis, including renal amyloidosis, glomerulonephritis, pyelonephritis, and malignant hypertension (Harrison et al, 1956). It is the purpose of this paper to report two cases of renal vein thrombosis, one complicating polyarteritis nodosa, the other the Henoch-Schoenlein syndrome, and to indicate a possible factor in the production of venous thrombosis in these conditions.

\section{CASE REPORTS}

CASE 1: POLYARTERITIS NODOSA The patient, a 16-yearold girl, had been fit and well until seven weeks before admission to hospital. She had then had a sore throat and this was treated with a sulphonamide. Four weeks before admission she developed muscle and joint pains, became febrile, and generally felt unwell. On admission she was pyrexial with an inflamed throat. Blood pressure was $110 / 70 \mathrm{~mm} \mathrm{Hg}$. The left ankle and the small interphalangeal joints were slightly swollen. Heart sounds were normal.

Investigations Haemoglobin was $12 \cdot 1 \mathrm{~g} / 100 \mathrm{ml}$, WBCs 8,500 , ESR $23 \mathrm{~mm} / \mathrm{hr}$, LE cells $\times 6$-negative, antistreptolysin titre 120 units, blood urea $18 \mathrm{mg} / 100 \mathrm{ml}$. In the urine a trace of protein and moderate numbers of red blood cells were found. The urine was sterile on culture.

A diagnosis of rheumatic fever was made but salicylates and penicillin produced no improvement. Five weeks after admission, thrombosis of the right saphenous vein occurred and a haemorrhagic indurated rash appeared over the elbows and feet. A diagnosis of polyarteritis nodosa was now thought to be most likely but

Received for publication 3 October 1968 skin and muscle biopsy showed no evidence of arteritis. She was then treated with prednisone, $30 \mathrm{mg}$ daily, wit improvement in her condition.

Seven weeks after admission she became pyrexial again with marked tenderness in the right iliac fossa and neutrophil leucocytosis of 30,000 per cmm. At laparo:tomy, petechial haemorrhages were seen over the wholf length of the colon and patchy thickening and reddening of the wall of the terminal small intestine were seen. Thछ appendix looked normal, but showed arteritic lesions when examined microscopically (Fig. 1). Following this operation the blood urea level gradually rose over the next three weeks to $300 \mathrm{mg} / 100 \mathrm{ml}$. She continued tQ have abdominal pain and low-grade pyrexia. Proteinuria became more marked, up to $3 \mathrm{~g} / 24$ hours, but the urinet volume remained above 1 litre daily. Nearly four weeks. after the operation and about 11 weeks after her admis sion to hospital she developed generalized convulsion? and died within a few hours.

Postmortem findings The main findings were multiple venous thromboses, infarcts in the kidneys and spleen haemorrhages into the mucosae and serosae of the gastrointestinal tract and into the subcutaneous tissues? There was pulmonary oedema, pleural effusions and peripheral oedema, and purulent peritonitis. A perio toneal swab taken at necropsy grew a coagulase-positive staphylococcus resistant to penicillin and tetracycline. of

All the large and medium size arteries appeared normal. Bilateral renal vein thrombosis had occurred both main renal veins being almost totally occluded, but the thrombus had not extended to the inferiof vena cava. The thrombus could be traced back as fao as the arcuate and interlobular veins. Thrombi werह్ also present in the left common iliac vein, right externa? iliac vein, and right saphenous vein. Pulmonary embolio were not present. The kidneys were pale and slightli enlarged (190 g each) but apart from a fairly recen⿷ infarct in part of the right kidney, they showed no focaf changes.

The appendix, removed four weeks before death showed arteritic lesions consisting of fibrinoid changes in vessel walls with a pleomorphic cell infiltrate of polyo 

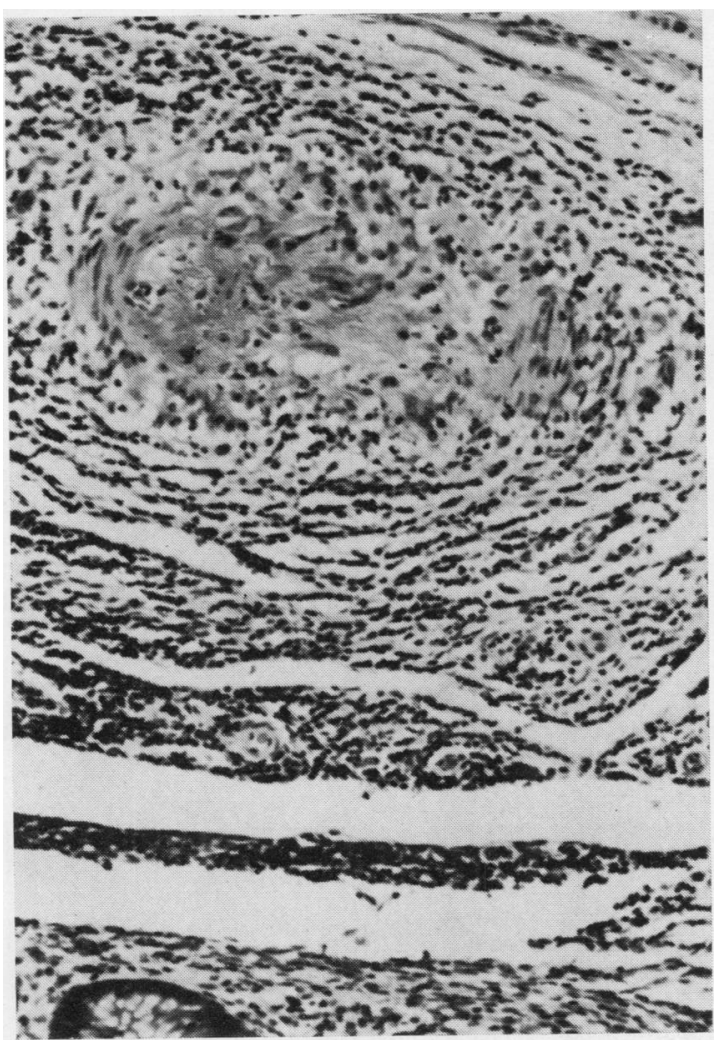

FIG. 1. Arteritic lesion in submucosa of appəndix (case 1). $\times 150$.

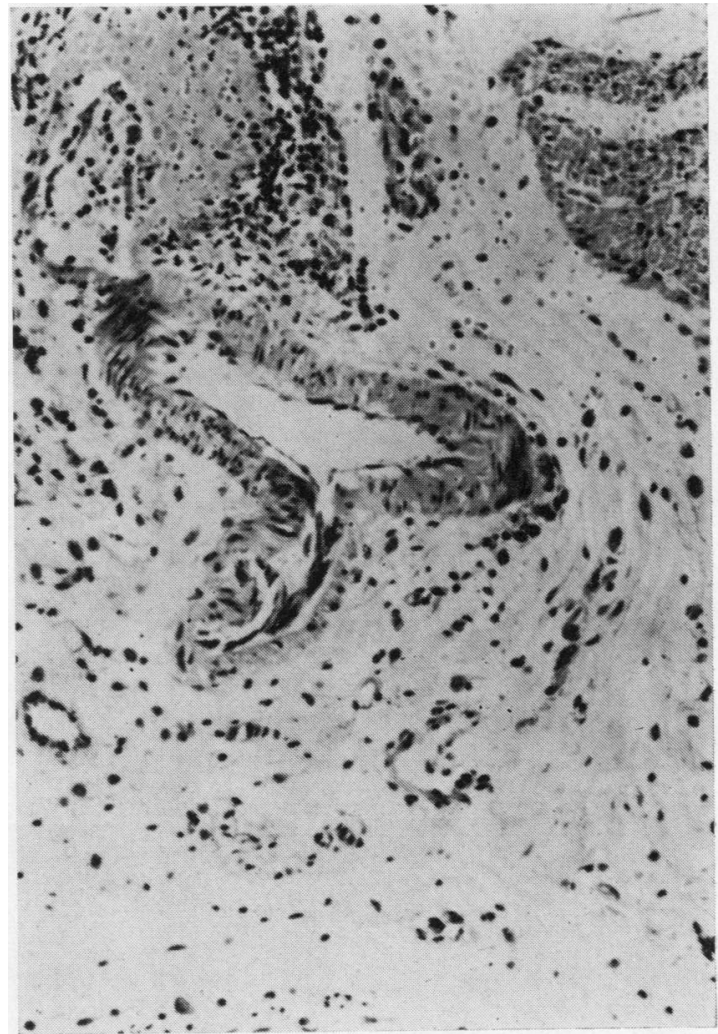

FIG. 2. Arteritic lesion involving part of arteriole wall in submucosa of small intestine (case 1$). \times 150$.

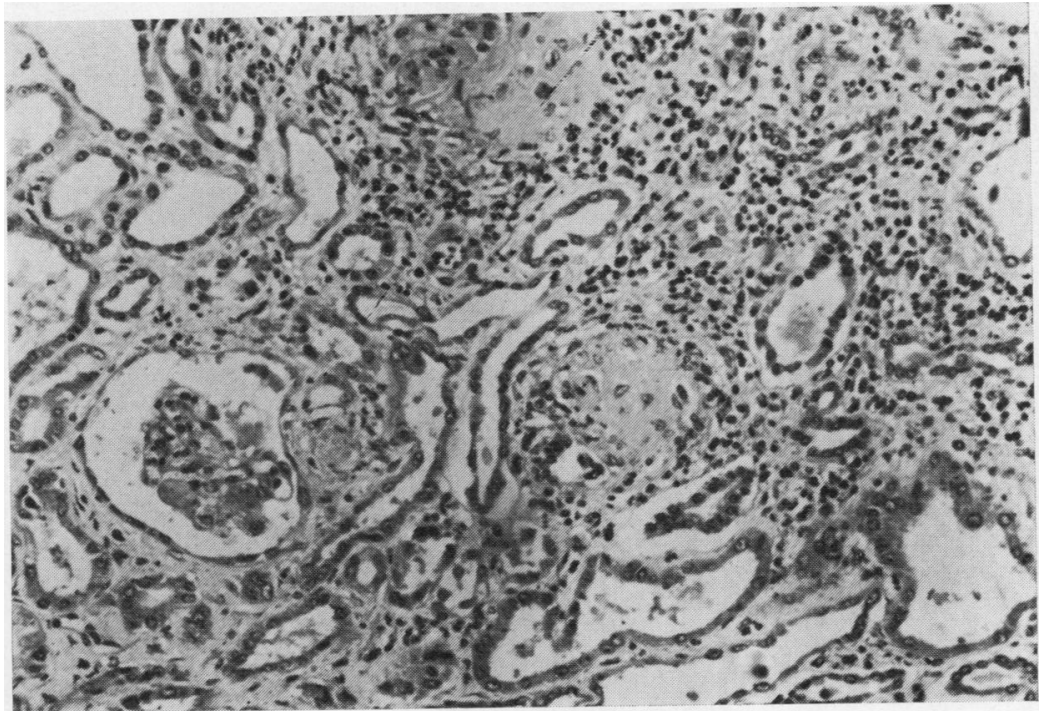

FIG. 3. Glomeruli showing different stages of involvement and one glomerulus partial necrosis (case 1). $\times 150$. 
morphs, eosinophils, and lymphocytes (Fig. 1). At necropsy similar arteritic lesions were present in the kidneys, gastrointestinal tract (Fig. 2), pancreas, and periadrenal fat. Vessels of between 100 and $500 \mu$ were mainly involved. Very gross changes were present in the kidneys. No normal glomeruli were seen, all stages from complete or partial glomerular necrosis to hyalinization being present (Fig. 3). A few epithelial crescents were seen. Interlobular arteries and smaller arterial vessels showed widespread arteritic lesions. The very extensive venous thrombosis was confirmed, the thrombi varying in age, although it was not possible to say whether the thrombotic process started in any one part of the renal venous system. In several places arteritic lesions were present in a small arteriole just exterior to the fibromuscular coat of a vein, in one instance in the wall of the main renal vein. The lesions stained negatively for amyloid stain (methyl violet).

CASE 2: HENOCH-SCHOENLEIN SYNDROME A 58-year-old woman was admitted with a 10-day history of abdominal pain and central upper abdominal tenderness. For the past six months she had had symptoms suggestive of

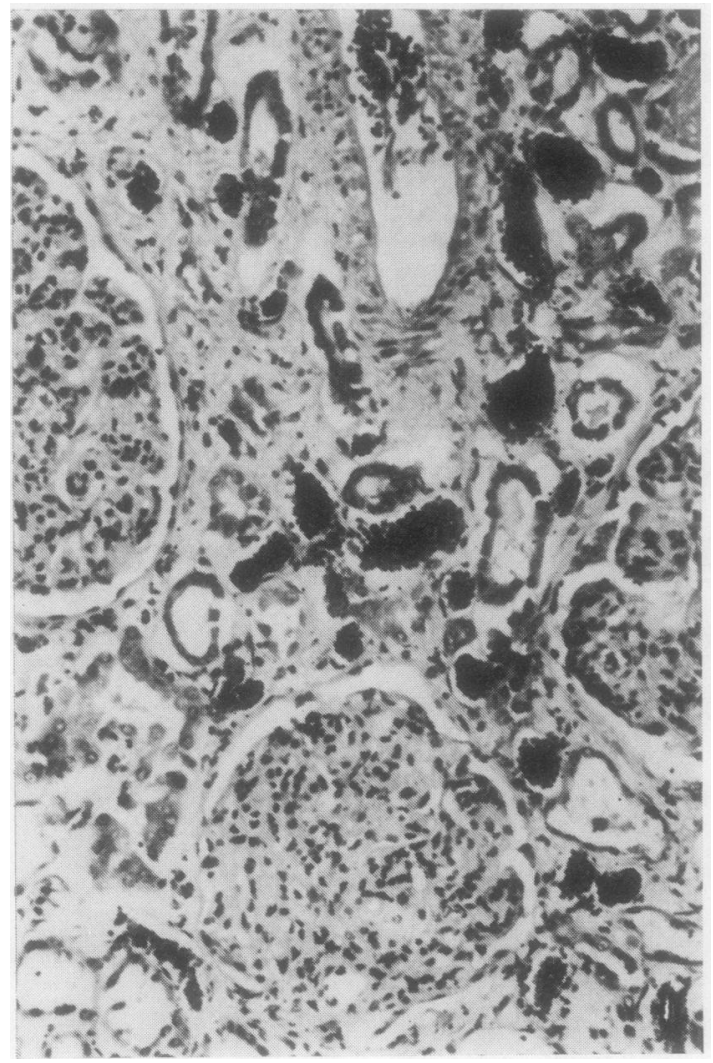

FIG. 4. Hypercellular glomeruli with adhesions to Bowman's capsule (case 2$). \times 150$. intermittent claudication. For the few days before admission she had noticed her urine to be dark.

Past medical history She had had recurrent bronchitis for seven years and mild urticaria and angioneurotion oedema for 20 years. On examination, blood pressure was $200 / 110 \mathrm{~mm} \mathrm{Hg}$ but there were no other abnormas signs.

Investigations A moderate amount of protein an moderate number of red cells were present in the urine $\propto$ The blood urea level was $36 \mathrm{mg} / 100 \mathrm{ml}$. Plasma proteins showed a rise in alpha ${ }_{2}$ globulins. The cholesterol leves was $250 \mathrm{mg} / 100 \mathrm{ml}$, ESR $50 \mathrm{~mm} / \mathrm{hr}$ (Westergren): haemoglobin and white cell count were normal.

The epigastric pain persisted and three weeks aftee? admission the patient developed the characteristic ras of the Henoch-Schoenlein syndrome. This started as a maculo-papular rash on buttocks and extensor surfaces of the limbs which later became purpuric with some् surrounding erythema. The Hess test was positive ando the platelet count normal.

Hypertension persisted and four weeks after admission? the blood urea level became raised and rose steadily reaching $250 \mathrm{mg}$ before death. Proteinuria became more

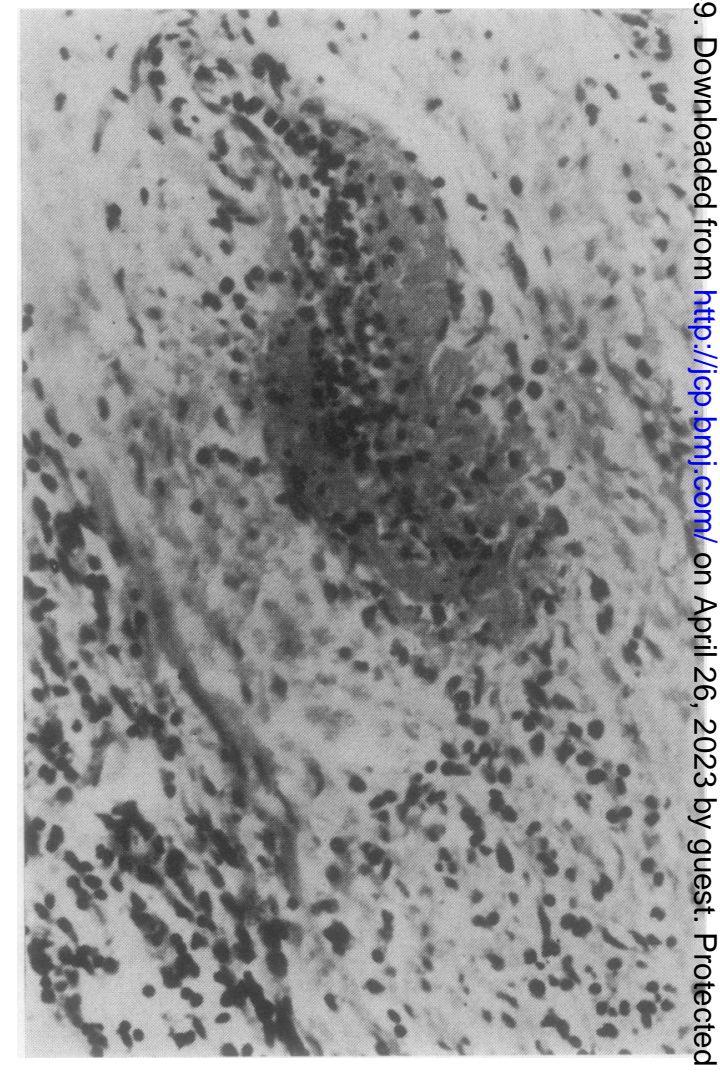

FIG. 5. Arteriole in the renal pelvis, showing fibrinot change and pleomorphic cell infiltration (case 2): $\times 200$ 
marked and for the last three weeks of life measured $12 \mathrm{~g} / 24$ hours.

During the last two weeks of life she developed widespread oedema of the face, arms, and legs, together with ascites and a left pleural effusion. Ten weeks after admission she developed generalized convulsions and died later the same day.

Postmortem findings The main findings were petechial haemorrhages into the skin, myocardium, and terminal ileum, and a small superficial haemorrhage over the left parietal lobe. Large and medium-sized arteries showed slight atheromatous changes but no other abnormality. No venous thromboses were noted. There was a peptic ulcer, $4 \mathrm{~mm}$ diameter, in the first part of the duodenum. The kidneys were pale and weighed $180 \mathrm{~g}$ each, the left kidney showing a small infarct. No other focal changes could be seen in the kidneys, the subcapsular surfaces being smooth.

Microscopic studies were unfortunately limited and no sections were available from the skin or gastrointestinal tract. The kidneys showed somewhat hypercellular glomeruli with adhesions to Bowman's capsule. Focal glomerular lesions were not seen and no crescents were present (Fig. 4). Arterioles in the medulla and renal pelvis (Fig. 5) showed an arteritis with fibrinoid change and a pleomorphic cell infiltrate composed of polymorphs, lymphocytes, and eosinophils. Several of the affected arterioles were seen in the adventitia of vein walls. Many medium-sized veins in the renal venous system were thrombosed but the main renal veins were not involved. Staining for amyloid was negative (methyl violet).

Arteritic lesions were also present in the lungs and right atrium, and in all areas examined the arterioles affected were less than $200 \mu$ in diameter. Small patchy areas of infarction were seen in the muscle of the right atrium.

\section{DISCUSSION}

Two other cases of renal vein thrombosis complicating polyarteritis nodosa have been recorded (Miller, Hoyt, and Pollock, 1954; Mandelbaum, Aftalion, Brody, and Hoffman, 1965). Renal vein thrombosis has not been noted in the HenochSchoenlein syndrome although the nephrotic syndrome may occur (Derham, and Rogerson, 1956). Renal vein thrombosis frequently produces the nephrotic syndrome and this was seen in case 2 .

The possible mechanisms for the production of the intrarenal type of venous thrombosis have been discussed by Barclay, Cameron, and Loughridge (1960). The main factors they considered were dehydration, raised fibrinogen levels, altered fibrinolytic activity, and decreased renal blood flow. The particular vascular structure within the kidney means that the obliterative lesions of the glomerular arterioles and capillaries (seen in the present cases) will reduce the blood flow in some areas of the kidney and lead to some degree of stasis on the venous side. It seems possible, also, that a thrombus once formed may tend to be self propagating. A further factor may be involved, as is suggested by these two cases. Veins throughout the body are richly supplied by small arteries (Bloom and Fawcett, 1962) and it is reasonable to suppose that the fully oxygenated blood they carry plays an essential role in the nutrition of the vein wall. In both these cases, small arteries in the vein walls in the renal pelvis were seen to be obliterated by the arteritic process (Fig. 6). It is suggested that the ischaemia brought about by the obliteration of these arterioles may induce changes in the venous endothelium, causing a thrombus to form at that point. This may have been one of the factors producing renal vein thrombosis in both these cases as well as the more widespread peripheral venous thromboses in the first

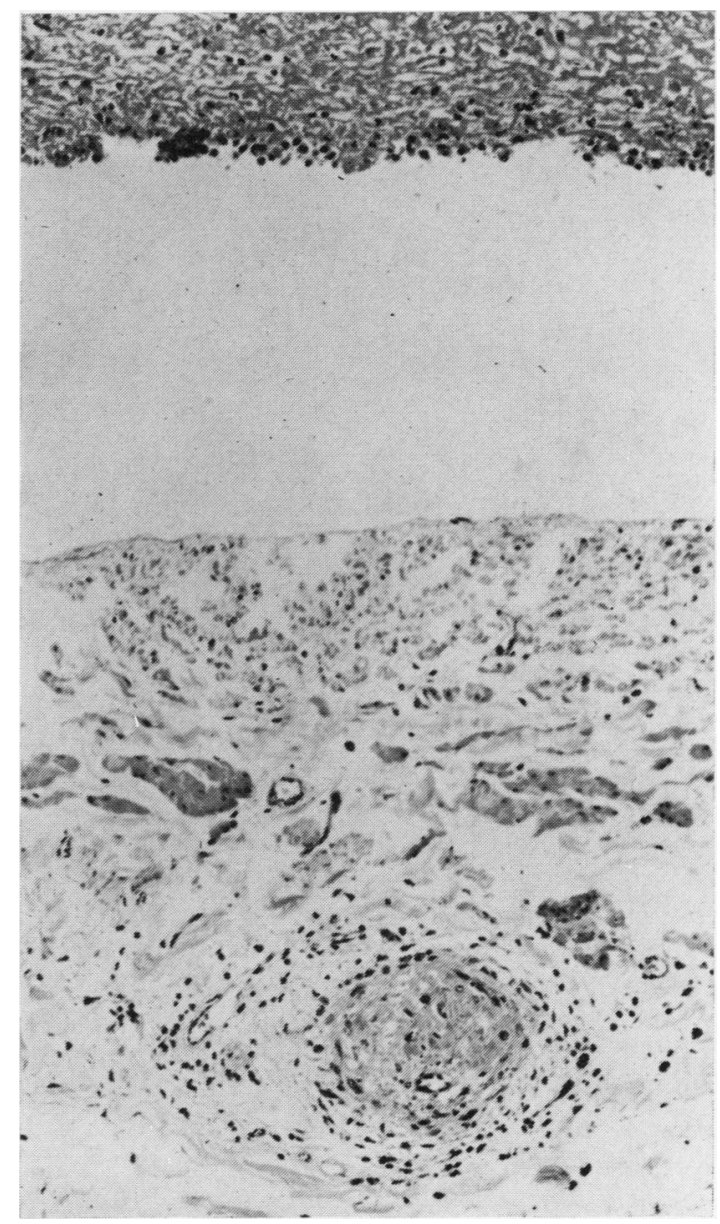

FIG. 6. The wall of the main renal vein from case 1 showing a healing arteritic lesion. The thrombus seen in the upper part of the photograph is attached to the vein wall at several adjacent points. 
case. Renal vein thrombosis of intrarenal type is probably easily missed at necropsy unless it is massive and involves the main renal veins. If it is specifically looked for, it may be found to occur more frequently in these conditions.

We are grateful to Dr F. Dudley Hart for permission to publish case 1 , and Dr P. Harvey for permission to publish case 2. We wish to thank Dr A. G. Signy for help in preparing this report.

\section{REFERENCES}

Barclay, G. P. T., Cameron, H. M., and Loughridge, L. W. (1960)으 Quart. J. Med., 29, 137.

Bloom, W., and Fawcett, D. W. (1962). A Text Book of Histology. 8 th ed. p. 281. W. B. Saunders Company, Philadelphia and London.

Derham, R. J., and Rogerson, M. M. (1956). Arch. Dis. Childh.ত $31,364$.

Harrison, C. V., Milne, M. D., and Steiner, R. E. (1956). Quart. J. Med., 25, 285. Mandelbaum, J., Aftalion, B., Brody, C. P., and Hoffman, I. (1965)
N.Y. St. J. Med., 65, 1790 .

Miller, G., Hoyt, J. C., and Pollock, B. E. (1954). Amer. J. Med.17,856 . 